IP Periodica Polytechnica Civil Engineering

\author{
59(4), pp. 591 601] 2015 \\ DOI: $10.3311 /$ PPci.8433 \\ Creative Commons Attribution (i) \\ RESEARCH ARTICLE
}

\section{Perspective with Six Vanishing Points - an Alternative Method}

\author{
Ágnes Urbin, Brigitta Szilágyi
}

Received 23-07-2015, revised 23-09-2015, accepted 24-09-2015

\begin{abstract}
The purpose of this paper is to describe perspective with six vanishing points. The line opened by axonometry and continued by one, two, three vanishing points and spherical perspectives, one became complete with the sixth vanishing point. Linear perspectives are often used, well known systems. Introducing new vanishing points doubles the represented part of space. The question of representing the half-space, hence the definition of the first five vanishing points can be found in literature. However, there was no useful solution for the representation of the whole space for a long time. This paper introduces a new definition of the sixth vanishing point that resulted a well applicable system. A short historical introduction looks at some of the interactions between mathematics and arts of western culture. Afterwards we formulate the new alternative perspective and give some methods of construction for notable lines and planes. Finally we write about possible applications.
\end{abstract}

\section{Keywords}

linear perspective $\cdot$ curvilinear perspective $\cdot$ vanishing point . space representation

\section{Ágnes Urbin}

Department of Mechatronics, Optics and Mechanical Engineering Informatics, Faculty of Mechanical Engineering, Budapest University of Technology and Economics, Múegyetem rkp. 3, H-1111 Budapest, Hungary e-mail: urbin@mogi.bme.hu

\section{Brigitta Szilágyi}

Department of Geometry, Faculty of Natural Sciences, Budapest University of Technology and Economics, Múegyetem rkp. 3, H-1111 Budapest, Hungary e-mail: szilagyi@math.bme.hu

\section{Historical introduction}

The first person we should mention about the history of perspective is al-Haytham. He contributed around 1000 A.C. in the medieval times. He was the first one who wrote about a concept that vision is the phenomenon when light is reflected from an object into the eye. Many of the artists we will mention in our introduction used his study of vision called perspective.

It seems that the antic Hellenistic painters did not understand the scientific background of perspective systems. There is always a problem: when we paint a picture of anything in a three dimensional space, we lose one dimension, so we lose information about the original scene. Therefore the mathematical rules of representation are needed and help to understand and make realistic pictures.

The first rules came by Giotto in the 13th Century. He directed the lines towards the eye-level as they moved away from the view-point. This way the lines above the eye-level came downwards, the lines below the eye-level came upwards and also the lines coming from the left and right sides were directed closer and closer to the centre. His rules were no more precise than these indications so Giotto was still far from an exact mathematical explanation, but it is clear that he tried really hard to understand the logic of representation.

The artist who defined the exact formulation of linear perspective was Brunelleschi in about 1413. In his method he understood that all parallel lines which fit to a plane other than the plane of the canvas should converge to a single vanishing point. Furthermore he understood well the basic of scaling - relation between linear distances of the real view and the image on canvas.

The person who described an explanation about the process of perspective representation was Alberti. Later on, many works were written about the mathematical and artistic explanation of perspective. Piero della Francesca brought in some quite unusual subjects: arithmetic, algebra and geometry around 1450 .

Leonardo da Vinci developed mathematical formulas to compute the relationship between the distance from an eye to the object and its size on the intersecting plane that is a canvas on which the picture will be painted. He distinguished two dif- 
ferent types of perspective: artificial perspective, which was the way that the painter projects onto a plane which itself may be seen foreshortened by an observer viewing at an angle; and natural perspective which reproduces faithfully the relative size of objects depending on their distance. In natural perspective, Leonardo correctly claims, objects will be the same size if they lie on a circle centred on the observer. Then he looked at compound perspective where the natural perspective is combined with a perspective produced by viewing at an angle.

By 1500, however, Dürer took the development of the topic into Germany. He published Unterweisung der Messung mit dem Zirkel und Richtscheit in 1525, the fourth book which contains his theory of shadows and perspective

In 1636 Desargues published the short treatise La perspective, which contains only 12 pages. In this treatise, which consists of a single worked example, Desargues sets out a method for constructing a perspective image, without using any point lying outside the picture field. He considers the representation in the picture plane of lines which meet at a point and also of lines which are parallel to each another. In the last paragraph, he considered the problem of finding perspective image of a conic section.

In 1719 Brook Taylor gives the first general treatment of vanishing points. The main theorem in Taylor's theory of linear perspective is that the projection of a straight line is not parallel to the plane of the picture it passes through its intersection and its vanishing point.

In modern times we find an interesting example of curvilinear perspective that, as its starting point, uses the same idea as Leonardo, when he considered projection upon a sphere.

The twentieth-century painter, engraver, and professor of art Albert Flocon developed the idea of making a central projection upon a sphere further in collaboration with his teaching colleague André Barre. The sphere only became an intermediate stage, as Flocon generally kept to the tradition of using a flat picture plane.

$\mathrm{He}$ and Barre therefore needed a transformation that could map an image from a sphere to a plane, and chose the so-called Postel projection. They used five vanishing points. Four vanishing points are placed around in a circle and there is one vanishing point in the centre of the circle. [1-[3]

\section{The path to the sixth vanishing point}

In this section you can read a summary of perspectives recited from axonometry to perspective with six vanishing points. The aim of perspective is to understand the rules of vision and to apply them in representation. To make easy to understand our specification of perspectives let's place a rectangular cube in an arbitrary orthogonal coordinate system. Position the cube with its edges parallel to the axes. We will observe with one eye from the origin of this coordinate system, our other eye should stay closed until the end of this paper.

The purpose of this paper is to systemize the perspective space representation methods so here we prefer being consistent and systematic rather than being expressive and visual. Further we will explain, where we had to make such decisions and we will compare the advantages and disadvantages of the visual, classical systems and our systematic representation.

The reason, why axonometry is one of the most often used systems of the technological terms is the fact that the way it represents three dimensional objects in plane is easily understandable and gives us exact information about the represented scene. Since pragmatical axonometries are not deteriorated we can state that none of the foreshortenings are equal with zero and none of the directions of the axes are similar to each other.

Axonometry is very popular in mechanical representation because by knowing the value of the foreshortenings it is easy and trivial to find out the exact measures. The disadvantage of axonometry is that the relative position of objects in space is not evident. In art we can find several examples where artists used axonometry to show impossible scenes as if they were real ones. Fig. 1 shows M.C.Escher's lithograph titled Waterfall. The picture shows an impossible situation caused by the rules of axonometry.

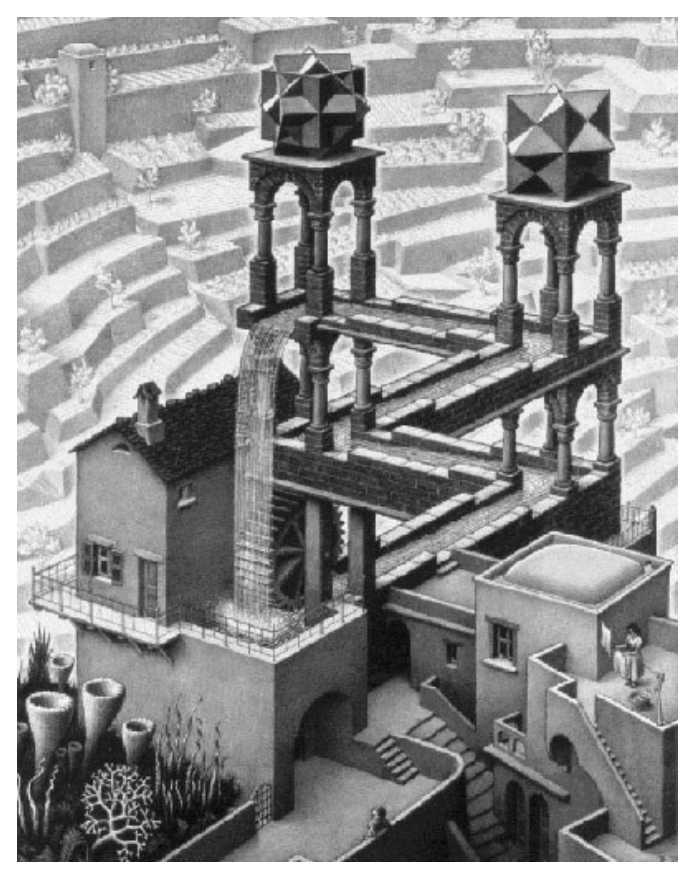

Fig. 1. M.C.Escher: Waterfall. The picture shows an impossible situation caused by the rules of axonometry

Leaving the homogeneous, parallel system of axonometry the aforementioned problem can be solved. If we represent foreshortening in one special direction, which is parallel to the direction of view, and project parallelly the planes perpendicular to the plane of the projection we practically use perspective with one vanishing point. The lines which are parallel to the direction of view converge to one point of the horizon: the intersection of the horizon and the viewing direction so the ideal point of all lines which are parallel to the direction of view.

Essentially perspective with one vanishing point is equal with Brunelleschi's linear perspective. This way we can represent the 
scene of a $30-40^{\circ}$ cone of view without significant distortion. See Fig. 2 for a simple example of the representation of a cube in perspective with one vanishing point.

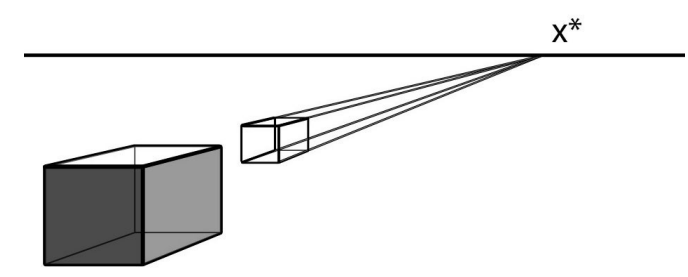

Fig. 2. Representation of a cube in perspective with one vanishing point

We can use one vanishing point if we would like to represent the interior of a room or building.

The disadvantage of this system is the problem first stated by Leonardo. He said the objects at equal distance from the plane of projection but at different distance from the spectator are represented in the same dimension. Fig. 3 shows as similar circles positioned on a line can be seen under different angles.

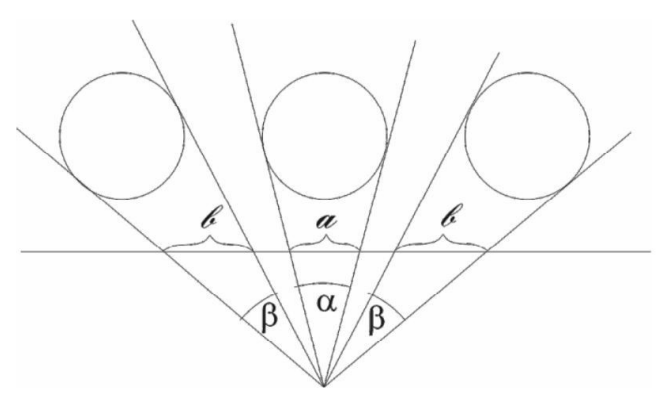

Fig. 3. Similar circles positioned on a line and seen under different angles

Basically that is why widening the cone of view in any direction causes disturbing distortion on the image and that is why the history of perspectives did not stop at this point. The more directions and with the bigger angles we widen the cone of view, the more directions we have to picture foreshortenings.

Let's define a linear scale on the horizon from $-180^{\circ}$ to $180^{\circ}$ in accordance with the direction of view so let be the origin the ideal point of the x axes: $O \equiv x^{*}$.

Take point $\mathbf{A}$ that can be seen at $\xi$ and point $\mathbf{B}$ that can be seen at $\eta$ from $\mathrm{x}$-axis. If neither of the points fit to $\mathrm{x}$-axis and $\mathrm{AB}$ segment crosses $\mathrm{x}$-axis we can count the angle at which the section defined by the two points can be seen. The line segment of $\mathrm{AB}$ can be seen at $(\xi+\eta)$ as shown on Fig. 4

What we know now is that straight lines parallel to $\mathrm{x}$-axis converge to a vanishing point and we also know that the lines perpendicular to the above mentioned lines do have a vanishing point. The section of these two points can be seen at $90^{\circ}$ which causes that we can exactly determine the relative position of the two vanishing points. Vanishing points are the ideal points of lines parallel to the axes of the predefined coordinate system. See Fig. 5 for a simple example of the representation of a cube with perspective with two vanishing points.

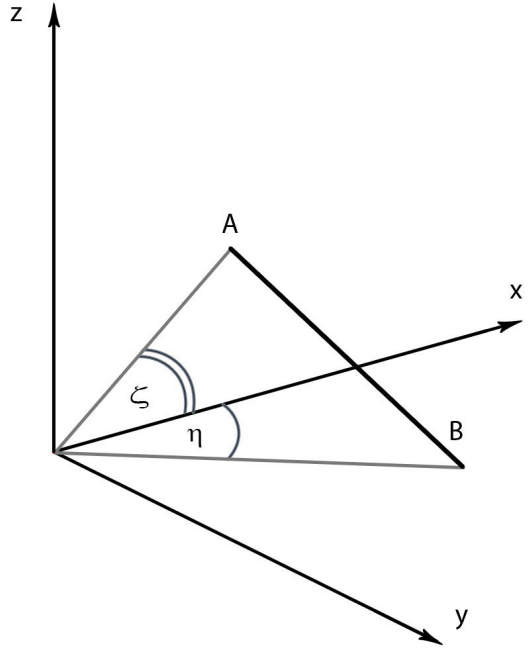

Fig. 4. The angle of view of $\mathrm{AB}$ segment

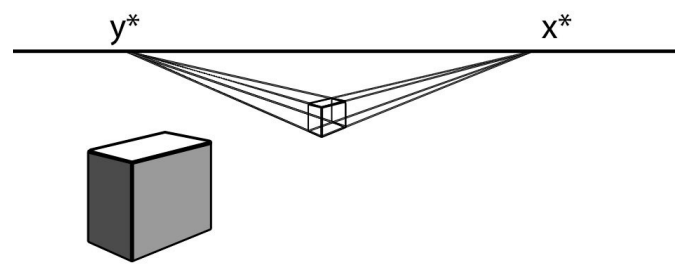

Fig. 5. Representation of a cube in perspective with two vanishing points

The perspective with two vanishing points is better than the one-point perspective if we want to give a truthful representation of a space from outside.

If the object is incomparable higher than the viewer, the threepoint perspective is useful.

As mentioned before, vanishing points are the ideal points of the axes of our cartesian coordinate system. When we set a new vanishing point we have to find the direction where the scene needs to expand the cone of view to prevent additional torsions. Since at the beginning the third vanishing point was used mostly in architectural representation, this usually belonged to the vertical (z) axis. This way after we position the first two vanishing points on the horizon regarding the best view of the original scene, we also have to decide if the third vanishing point should be set beyond or beneath the horizon. Plotting on paper this means that we have a horizontal section defined by the first two vanishing points (belonging to $\mathrm{x}$-axis and $\mathrm{y}$-axis in our coordinate system) as the distance between the two endpoints is $90^{\circ}$ of our above mentioned scale, and a vertical section which represents the $\mathrm{z}$ axis. This section can be seen also at $90^{\circ}$ and its connection to the horizon and its vertical orientation depends on the current represented scene and the observer's position.

Fig. 6and Fig. 7 show two examples for the representation of a cube with three vanishing points. As it can be seen changing the position of $\mathrm{z}$-axis causes changes in the distortion.

This way we represent ideal points of all three axis and we can picture the impression of the three dimensional space in a quite wide field of view. 


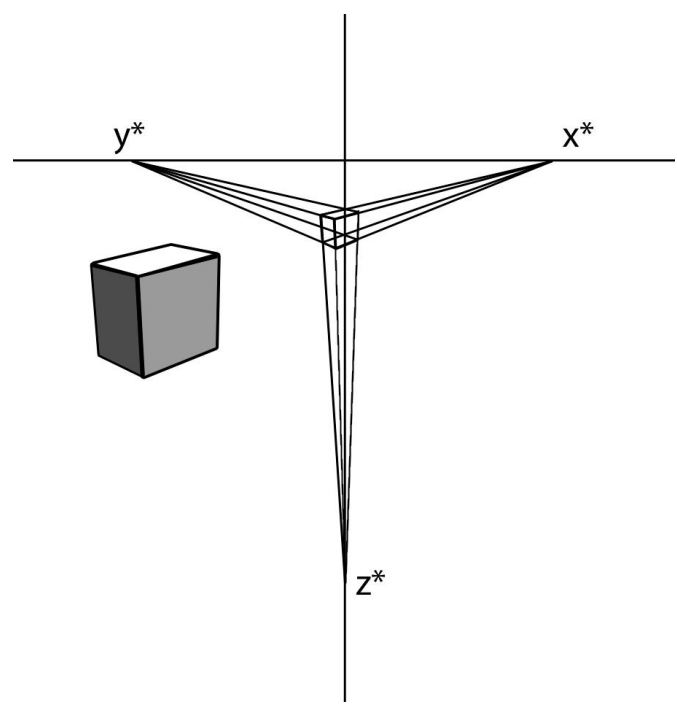

Fig. 6. Representation of a cube in perspective with three vanishing points in the classical system.

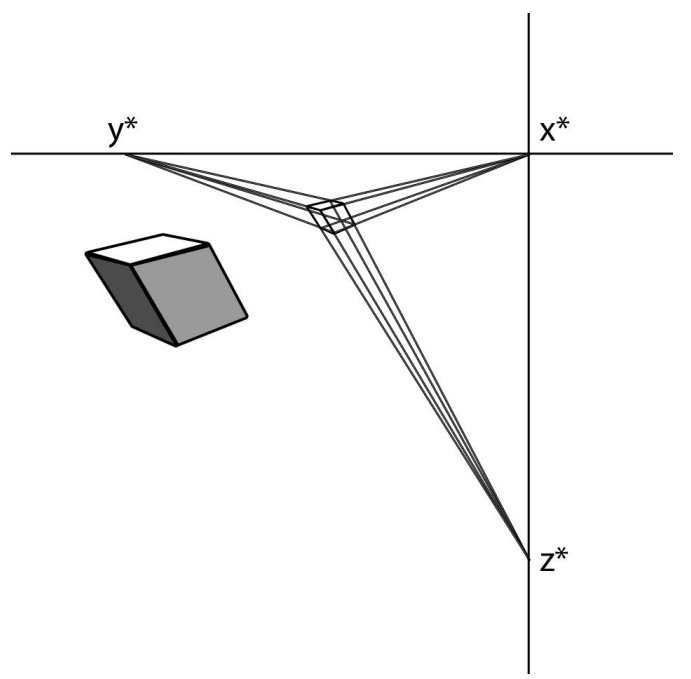

Fig. 7. Representation of a cube in perspective with three vanishing points in our system

The linear perspectives (one-point, two-point and three-point perspectives) are used when the objects are positioned inside a well-defined field.

Fig. 8 shows an explanation for the difference between space representation onto planar and spherical canvas.

Even so as we try to extend the field of view in any direction, sooner or later we have to face the problem that parallel lines seem to converge to different vanishing points which concludes to the fact that the picture of lines are not always linear. This contradiction can be solved understanding the following thoughts. However we represent ideal points of all three axes, we represent only one of each so we represent only half of those lines, actually halves of spaces in turn. If it is understandable that two parallel lines converge to an ideal point in front of us, the same has to happen also behind us. Consequently we can not ignore the second ideal points of our axes and the lines which are parallel to the axis of which we represent both ideal points must converge to both vanishing points. The section of two vanishing points of an axis can be seen at $180^{\circ}$ as shown on Fig. 9

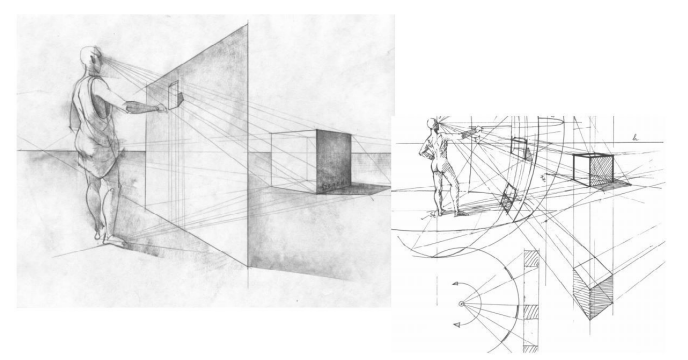

Fig. 8. Illustration of space representation where the canvas is planar (left) and spherical (right)

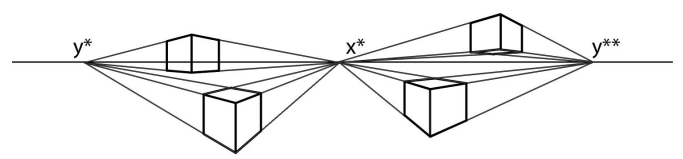

Fig. 9. The edges of the cubes are set parallelly to y-axis hence those edges shoud converge to $\mathrm{y}^{*}$ and $\mathrm{y}^{* *}$ also.

That is how we arrive to the perspective with four vanishing points. In the classical system in order to get the four-point perspective we need a half-pipe as image surface. This solution reflects in the famous panorama images of the $19^{\text {th }}$ century. However if we would like to create a unified definition of the curvilinear perspectives, the introduction of the fourth vanishing point should be as the followings.

Considering the relation of the axes we can appoint that installing the fourth (and each following) vanishing point we duplicate the representable part of space.

In the examples seen on Fig. 10 and Fig. 11 we represent both vanishing points of the vertical z-axis. Since this mode of representation is quite infrequent, there are no conventions, the set of vanishing points always depends on the actual composition. We refer to a plausible, symmetrical solution as classical system.

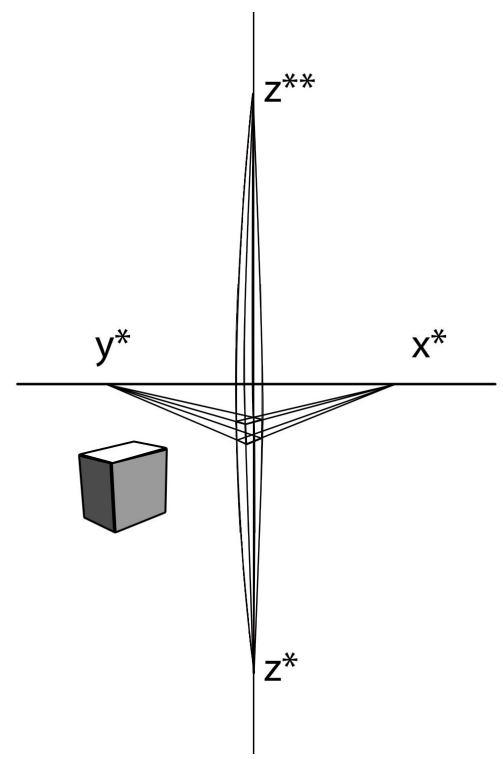

Fig. 10. Representation of a cube in perspective with four vanishing points in the classical system 


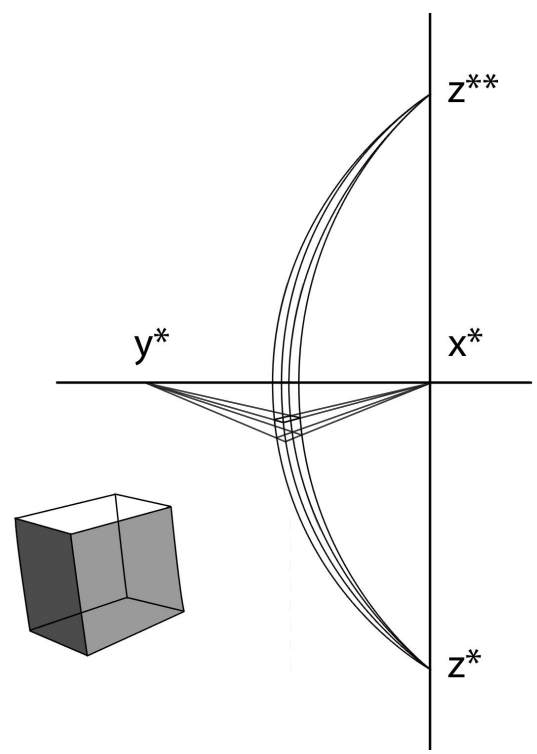

Fig. 11. Representation of a cube in perspective with four vanishing points in our system

Otherwise if we introduce the list of perspectives in a way that however we have not found in the literature but we found in applications we should be neglect the fourth vanishing point. [4]

Similarly to the fourth vanishing point, the axis belonging to the fifth vanishing point is also optional. Now we keep the ideal point of $x$-axis as front vanishing point (so the direction of view) and represent two-two vanishing points of $y$ and $z$ axes. If we work on the figure we used before, the fifth vanishing point causes a distorted picture as shown on Fig.12 (left). In order to show demonstrative picture we can move the intersection of the horizon and the image of the z-axis to the image of the vanishing point of the $\mathrm{X}$-axis. In this case the five vanishing points are positioned as shown on Fig. 12 (right).

The image surface is a hemisphere in this case so we map the half space in front of us as shown on Fig. 13

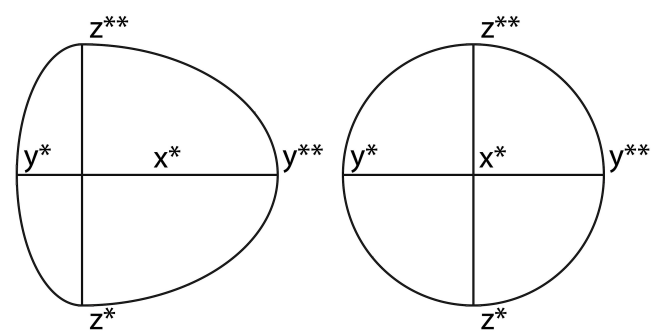

Fig. 12. Fields of representation in perspective with five vanishing points depending on the direction of view.

Maybe because of the unusual view caused by the distortions the curvilinear perspectives are less common. Compared to the centuries-long history of linear perspectives they appeared only in the last decades.

\section{The sixth vanishing point}

If we want to map the whole space around us we have to define the sixth vanishing point in a systematic way.

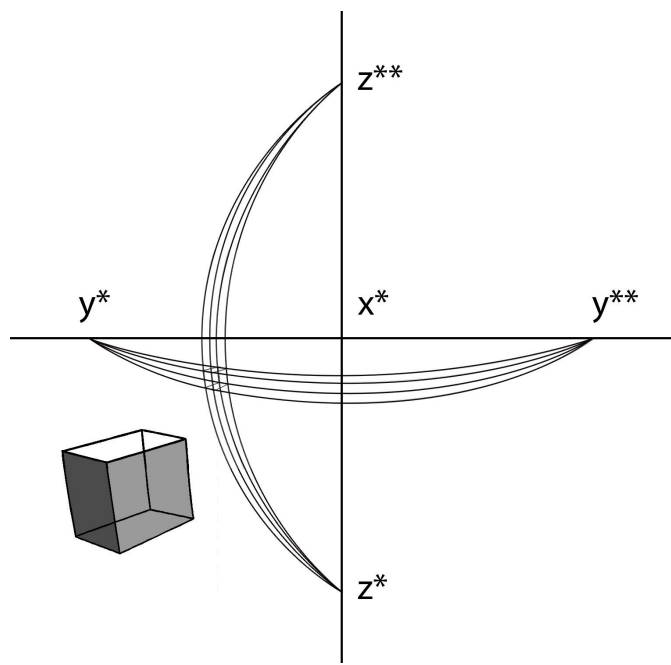

Fig. 13. Representation of a cube in perspective with five vanishing points

To create a system that can be well used in applications is not so easy. Maybe that's why corresponding literature is quite minimal; mostly artistic books and papers deal with this kind of space representation methods offering alternative solutions.

Reviewing the fine arts we can find many solutions of representing the whole space around us but most of these angle the graphical, perceptible representation just like the renaissance artists. There are some enterprising artists experimenting with this kind of methods primarily using their intuition but no scientific applications were found.

An artist, Dick Termes created a new method in 1968. He works on a special big ball so-called termeshpere. "Each Termesphere is a revolving three-dimensional space/time exploration of an entirely closed universe, meaning that what you see, rotating in front of you, is one complete world or environment." [5]

Attila Bölcskei gave a method of new visualisation of straight lines and planes that have similar fundamentals to perspective with six vanishing points. [6,7]

If we follow the logic of the previous chapter, we can define the sixth vanishing point as the ideal point of the third axis (in our case the $\mathrm{x}$-axis). However the concept is clear, the plotted picture of the sixth vanishing point is not obvious at all.

What do we know about the sixth vanishing point?

1 According to the deduction of the last chapter we know that the section of the vanishing point in front of us and behind us (namely the two ideal points of the $\mathrm{x}$-axis) can be seen at $180^{\circ}$.

2 If a point creates a section seen at $180^{\circ}$ with the vanishing point in front of us, then the point is situated exactly behind us and the image of this point is coincident with the sixth vanishing point.

The above mentioned conditions conclude to that the image of the sixth vanishing point is not a single point but a circle (as shown on Fig. 14). This circle has a radius of $180^{\circ}$ and centred at the origin, the image of the vanishing point in front of us. 
In order to make orientation easier we always represent with grey the image of the horizon, the vertical direction (z-axis) the image of the sixth vanishing point and the plane perpendicular to the direction of view and containing the origin.

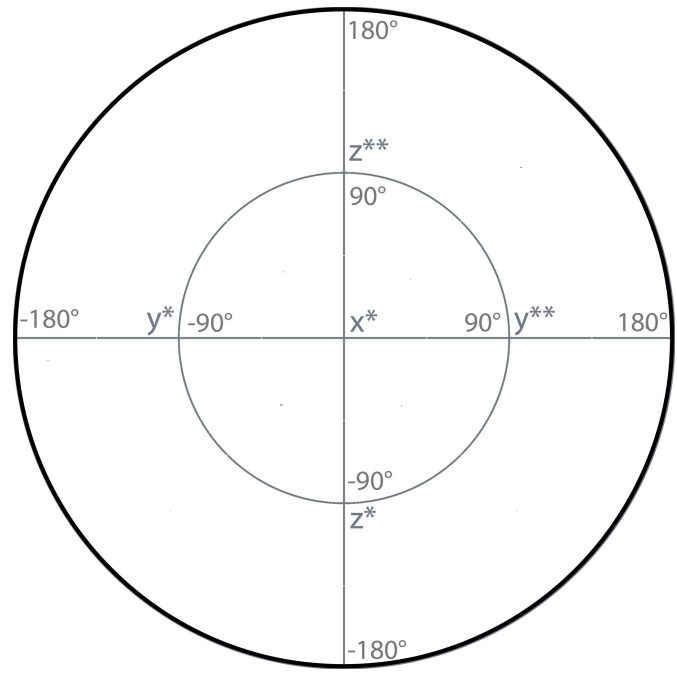

Fig. 14. The image of the sixth vanishing point

This circle defines the whole canvas in which we can represent a scene with this method. This field can be understood as a coordinate system.

In order to make this representation more than expression we have to find the rules: how does it really work?

We use two angles as variables of this representation method. Take point $\mathbf{P}$ with (x, y, z) coordinates in our cartesian coordinate system. We define $\alpha$ as the angle between the horizon (y-axis) and the normal projection of the OP vector (OP') and $\beta$ as the angle between the direction of view ( $\mathrm{x}$-axis) and the OP position vector. See explanation on Fig. 15

The correspondence between $(\mathrm{x}, \mathrm{y}, \mathrm{z})$ and $(\alpha, \beta)$ can be drawn with the following simple trigonometrical formulas.

$$
\begin{gathered}
\alpha=\operatorname{arctg} \frac{z}{y}, \text { where } y \neq 0 \\
\beta=\operatorname{arctg} \frac{\sqrt{\left(y^{2}+z^{2}\right)}}{x}, \text { where } x \neq 0
\end{gathered}
$$

Regarding the periodicity of the trigonometric functions we always have to consider in which eighth part of space we are and make small corrections. We can carry out similar calculations.

The $\alpha$ and $\beta$ values can be represented in a polar coordinate system as seen on Fig. 16 .

However this is less expressive than many artistic solutions, we have to take notice that representation with these six vanishing points gives us the chance to represent the whole space irrespectively of the direction of view. The image is defined only by the point of view. Once we have a picture from the selected point, we can change the view with basic transformations of the image. The transformations can be easily handled since the image will be set into a finite system in which the image of straight lines remain continuous (note the exceptions when the line fits to the origin).

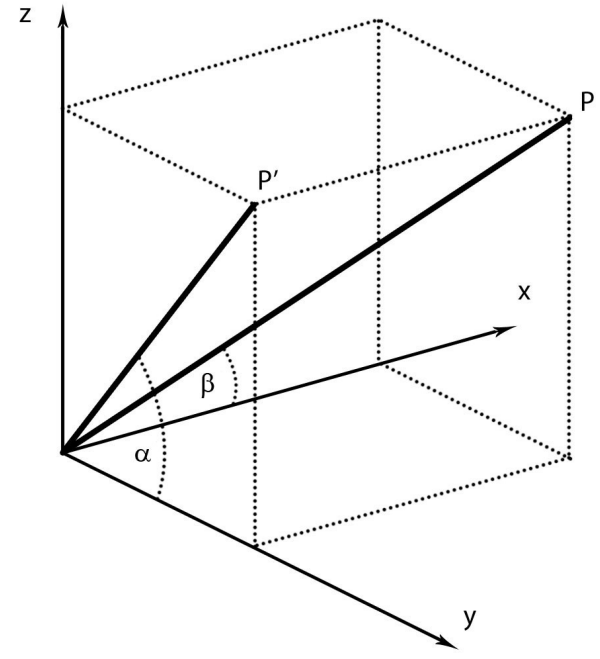

Fig. 15. $\alpha$ and $\beta$ coordinates of point $\mathbf{P}$

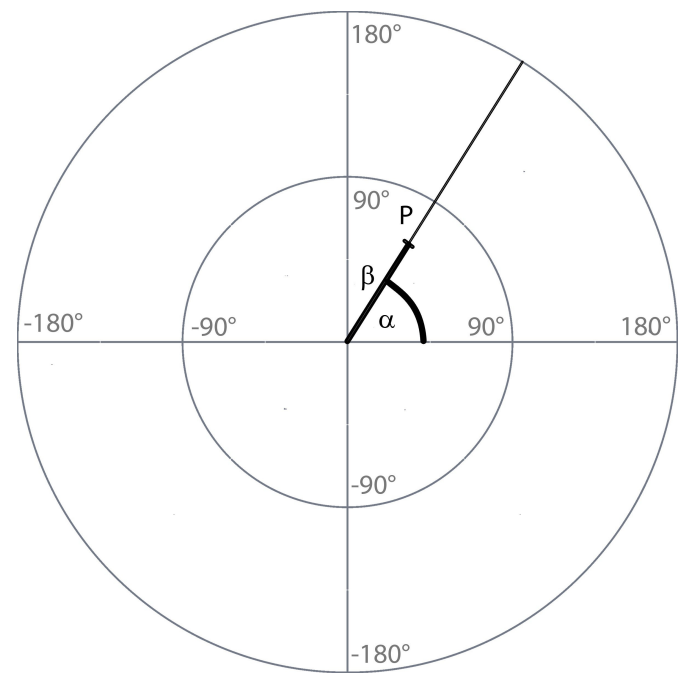

Fig. 16. Representation of point $\mathbf{P}$ in perspective with six vanishing points

Hipothesis: Define $f: \mathrm{R}^{3} \mapsto \mathrm{R}^{2}$ mapping as the followings:

$$
\left[\begin{array}{c}
x \\
y \\
z
\end{array}\right] \mapsto f(x, y, z)=\left[\begin{array}{c}
\alpha(y, z) \\
\beta(x, x, z)
\end{array}\right],
$$

where $\alpha(y, z)$ and $\beta(x, y, z)$ are defined as in Eqs. (1) and (2). This mapping satisfies the rules of perspectivic space representation and can be applied in practice.

Irrespectively of the consideration of the trigonometric periodicity we must make the following two restrictions: $x \neq 0$ and $y \neq 0$ since the denominators of Eqs. (1) and (2) can not be equal to zero. Note that these restrictions refer to cases that are quite easy to represent graphically. Mathematically the $\alpha$ and $\beta$ coordinates of these points can be calculated as the limits of the actual arctg functions close to the limits of the domains of definition.

In order to prove the mapping defined by us let's see the properties of the basic geometric objects and transformations.

Take an arbitrary set of points, for example a straight line in space. To know more about this we must get to know more about the features of this perspective. The first step of representation is 
similar to the central projection of the one, two or three centres but in this case we project the points onto a sphere while we observe from the centre of sphere. Next we project these points onto a plane with the above mentioned mapping.

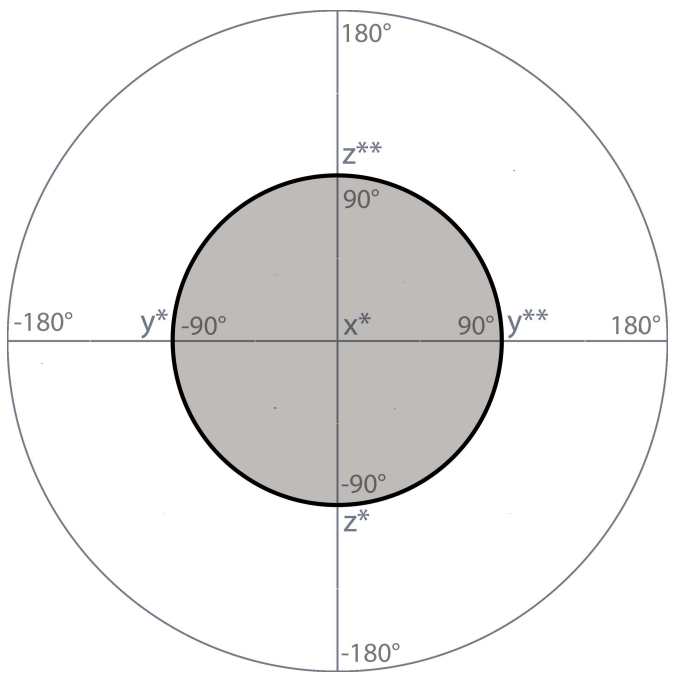

Fig. 17. Planes perpendicular to the direction of view in front of the observer

\subsection{Notable planes}

The planes in front of us which are perpendicular to the direction of view can be represented also in perspective with five vanishing points. $\alpha$ and $\beta$ values of the points of these planes can be the following: $\alpha \in\left[0^{\circ}, 360^{\circ}\right]$ while $\beta \in\left[0^{\circ}, 90^{\circ}\right]$. The values $\beta=0^{\circ}$ and $\beta=90^{\circ}$ belong to the corresponding limit cases. As shown on Fig. 17 these planes are represented in the inner circle of the field.

The planes behind us which are perpendicular to the direction of view have the same $\alpha$ value ( $\alpha \in\left[0^{\circ}, 360^{\circ}\right]$ ) but the $\beta$ value (where $\beta$ is the angle between the direction of view and the position vector of a represented point) is $\beta \in\left[90^{\circ}, 180^{\circ}\right]$. The values $\beta=90^{\circ}$ and $\beta=180^{\circ}$ belong to the corresponding limit cases. As shown on Fig. 18 these planes are represented in the outer circle of the field.

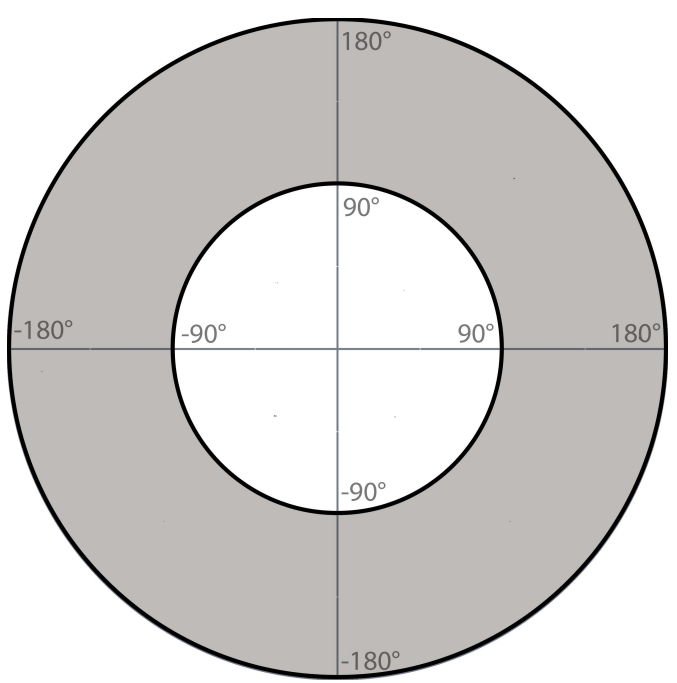

Fig. 18. Planes perpendicular to the direction of view behind the observer

\subsection{Notable lines}

Studying the lines parallel to the direction of view we noticed that every straight line has a constant $\alpha$ value and to all $\alpha$ it assume all $\beta$ values $\left[0^{\circ}, 180^{\circ}\right]$. The value $\beta=0^{\circ}$ belongs to the corresponding limit case. This means that the images of these straight lines remain also straight and will be a radius of the circle (see Fig. 19-e). The image of lines that are parallel to $y$ - or z-axis became curvilinear (see Fig. 19- f). The specific points of these curves are the endpoints (the vanishing points of the actual axis) and the point where the curve crosses the axis perpendicular to the actual one and to the direction of view. The image of those straight lines which contain the origin will be the two ideal points of the line (see Fig. 19- $\mathrm{g}$ ).

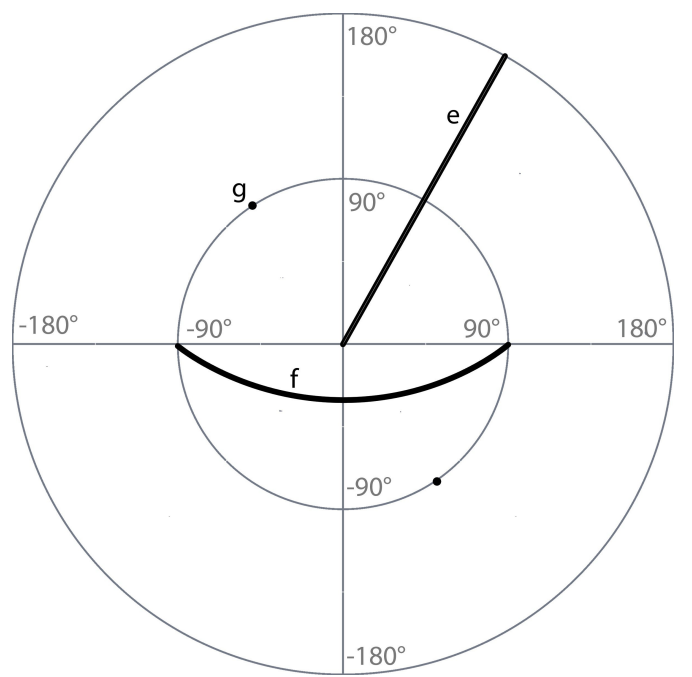

Fig. 19. Notable lines: parallel to the direction of view (e) parallel to y-axis (f) and crossing the origin (g)

\subsection{Reflection}

Take point $\mathbf{P}$ with known $\alpha$ and $\beta$ values and reflect this point onto the plane perpendicular to the direction of view and containing the origin. Thus $\mathbf{P}$ is moving along a line parallel to the direction of view $\alpha$ values of these two point are the same. Besides due to the features of the reflection $\beta^{\prime}=180^{\circ}-\beta$. (see Fig. 20,

\subsection{Rotation}

In case of the plane which is perpendicular to the direction of view and contains the origin $\beta=90^{\circ}$ belongs to all $\alpha$ values. It is notable that the image of each plane containing the origin is a closed curve.

Rotate this plane around z-axis with $\varphi$ (in our example $\varphi=45^{\circ}$, see Fig. 21). Now we know that this plane still contains the vanishing points marked by z-axis. We also know the angles that belong to $\alpha^{\prime}=0^{\circ}$ and $180^{\circ}$.

$$
\begin{gathered}
\text { If } \alpha^{\prime}=0 \text { then } \beta=\beta-\varphi \text {. } \\
\text { If } \alpha^{\prime}=180 \text { then } \beta=\beta+\varphi,
\end{gathered}
$$

where $\alpha^{\prime}$ and $\beta$ ' denote $\alpha$ and $\beta$ values after the rotation. 


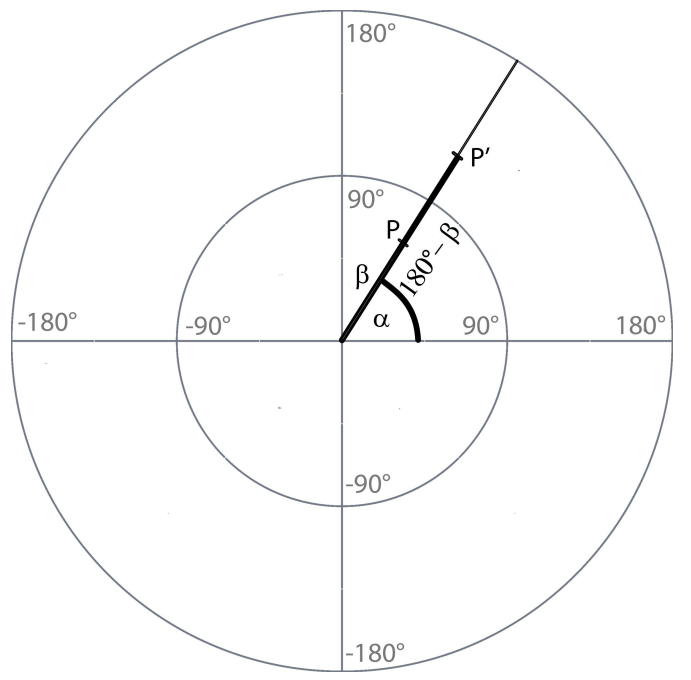

Fig. 20. Reflection of $\mathbf{P}$ onto the plane perpendicular to the direction of view and containing the origin

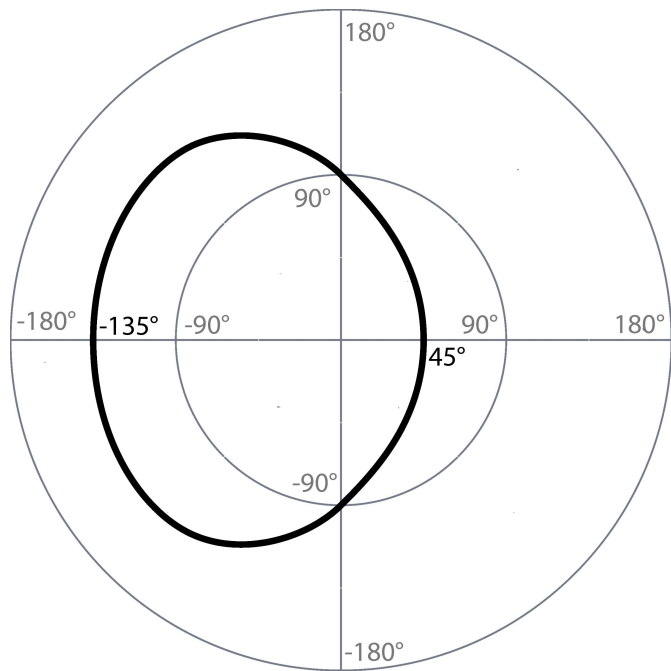

Fig. 21. The image of a plane crossing the origin and rotated with $45^{\circ}$ around z-axis

By knowing the above mentioned features we can already describe basic geometric solids not only point by point, but also with the help of the planes and the plane surfaces bordering them.

As an example let's take a cube which is examined from the intersection of its diagonals, so that our direction of view is normal to ABCD plane. Fig. 22 shows the image in axonometry and Fig 23 shows the same cube in perspective with six vanishing points.

We know that ABGH points are on the plane, which we get by rotation the plane normal to the direction of view by around z-axis. With a similar transformation we can draw the picture of the planes defined by the BCHE, CDEF and DAFG rectangulars. The intersections of these planes define the place of the vertices of the cube in space, and the intersections of the curves defining the images of the planes set the places of the vertices in the plane.

AE, BF, CG and DH edges are parallel to the direction of view so we know that their images will be lines heading for the

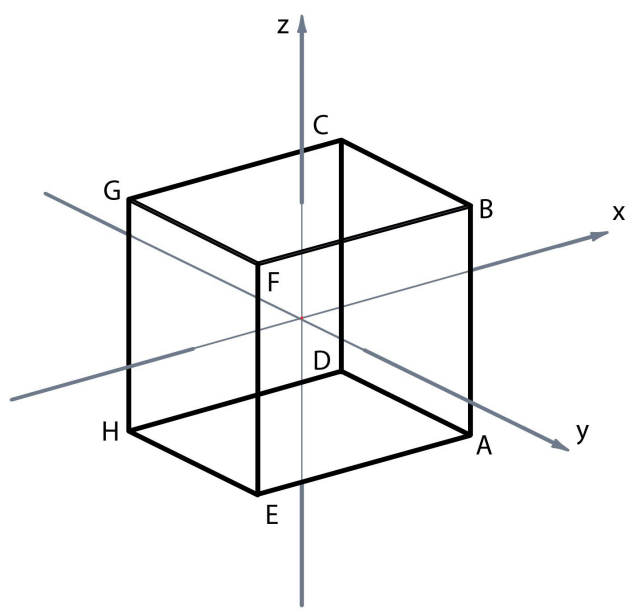

Fig. 22. Image of a cube in axonometry

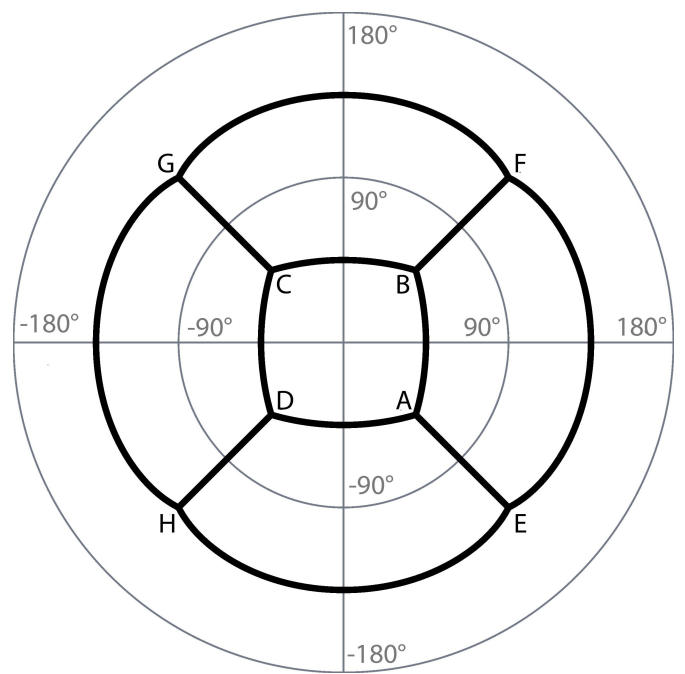

Fig. 23. Image of a cube in perspective with six vanishing points observing from the intersection of its diagonals towards the ABCD plane

origin and also knowing the geometric features of the cube we can realize that the $\alpha$ values belonging to the four edges are $\pm 45^{\circ}$ and $\pm 135^{\circ}$.

\section{Applications}

Assuming the possibilities of the practical use of this perspective at first sight it can seem to be an uncommon space representation method and no more. Actually there is much more. Understanding this method can help to solve conceptual problems that hinders to consider perspective both from artistic and scientific point of view. In the renaissance ages it was evident to research this topic from both aspects since neither the disciplines were separated as nowadays.

Besides there are mentionable technical application possibilities also.

\subsection{Computer visualisation}

The advantages of this system is that with the above mentioned equations it is easy to handle digitally. The first steps made by us were some simple programs to show the steps of this representation. 
Fig. 24 shows the steps of rotation of planes from $[y, z]$ to plane $[\mathrm{x}, \mathrm{z}]$.

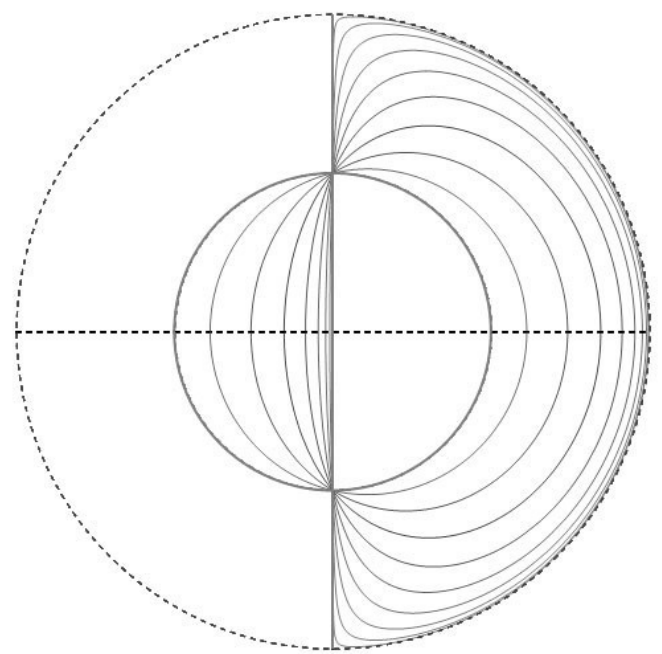

Fig. 24. Rotation of planes from $[y, z]$ to $[x, z]$

Fig. 25 shows the steps of rotation from plane $[\mathrm{x}, \mathrm{y}]$ to plane $[\mathrm{X}, \mathrm{Z}]$.

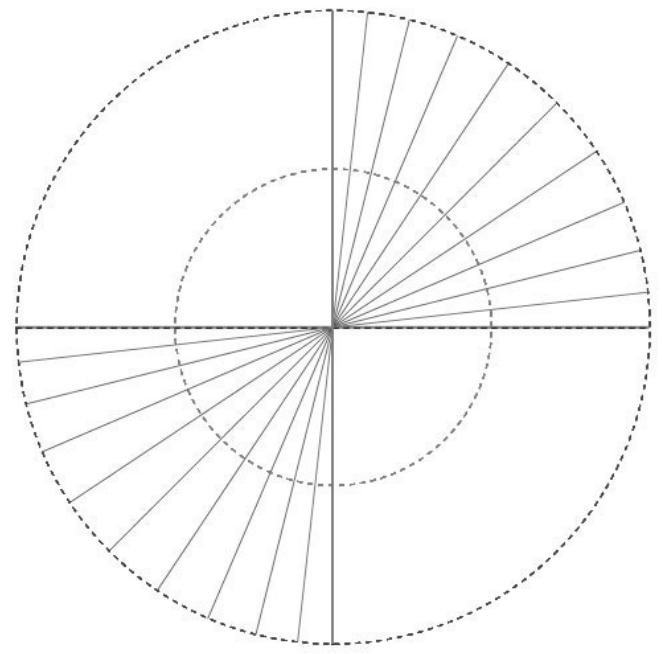

Fig. 25. Rotation of planes from $[\mathrm{x}, \mathrm{y}]$ to $[\mathrm{x}, \mathrm{z}]$

Fig. 26 illustrates a dodecaeder in axonometry and perspective with six vanishing points. As it can be seen, the image close to the direction of view is quite similar to the visual world we are used to.

With this way of representation the relation of the symmetries can be explained and detected easily.

A possible 2D application is to create a fish-eye effect on a picture captured by a camera with normal angle of view. Two dimensional image editor softwares already give solution to this problem but the solutions give spectacularly inaccurate results. Fig. 27, Fig. 28 and Fig. 29 show the aula of the main building of the Budapest University of Technology and Economics with different representation methods. Each image was created from the first floor and the direction of view was the same.

Fig. 27 was created from a picture with wide angle of view distorted with a software to imitate fish-eye effect. Inaccuracy
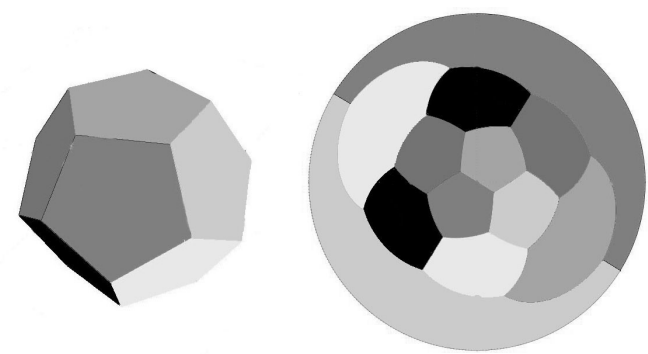

Fig. 26. A dodecaeder represented in axonometry and in perspective with six vanishing points

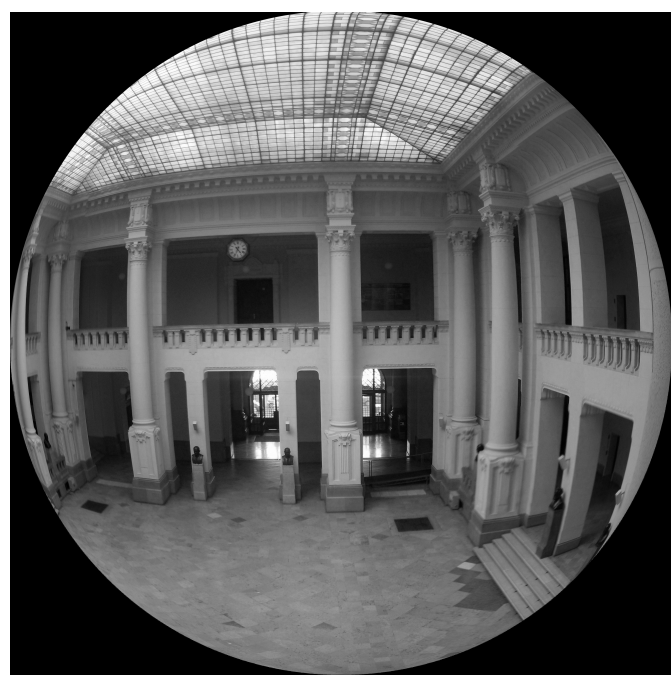

Fig. 27. The aula of the main building of BME represented with wide viewing angle and distorted to imitate fish-eye effect.

can be detected on the ceiling where the grid parallel to the direction of view should converge to the main vanishing point defined by the direction of view.

Fig. 28 was created with fish-eye effect from the same position than Fig. 27. As it can be seen a much wider part of the space could be represented however the pillar on the right covers the main part of the corridor on the right. Eventually the image should have been taken from the middle of the pillar but technically that was not possible in our case.

Fig. 29 was created by an Architect student, Zsofia Helmeczi during a course about the rules of perspective with six vanishing points. The exercise was to draw the aula of the main building based on the ground plan and the side-views. The direction of view was the same than on Fig. 27 and Fig. 28 but the point of view was not fixed to the loft, it was defined to be in the same distance from the pillar towards and backwards the direction of view. Basically as if the observer floated close to the middle of the aula.

Understanding the rules of the representation and the layouts students could draw this quite complex space as if they were sitting in the middle of the aula vertically. This way even though the image of the objects behind the observer is not expressive, this image gives unique connection to the layout of the aula.

This course showed that this method could be used in education also. Using this method gives an approach to understand spaces in another way than as it is taught now. 


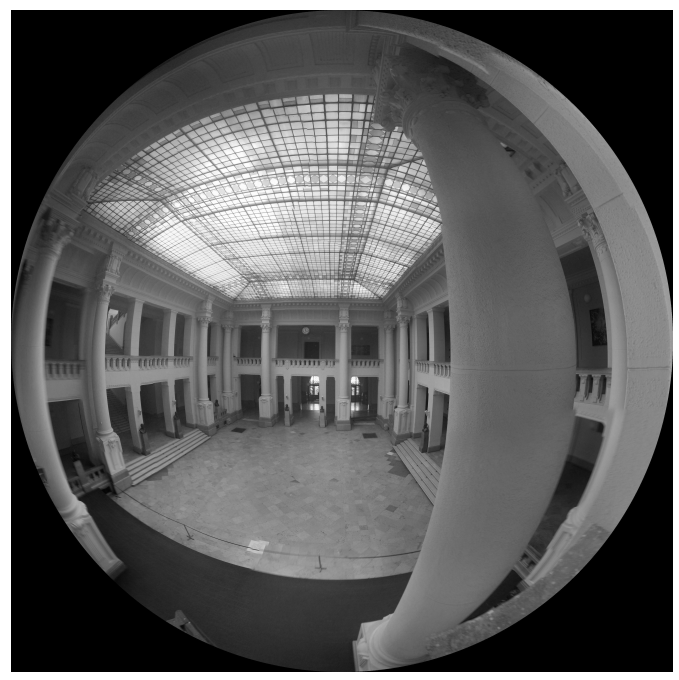

Fig. 28. The aula of the main building of BME represented on a photo with fish-eye effect

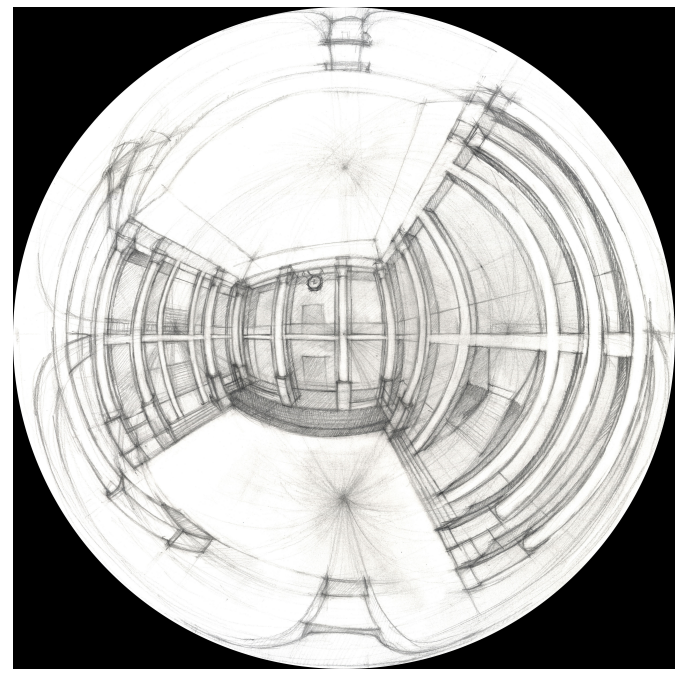

Fig. 29. The aula of the main building of BME represented in perspective with six vanishing points.

Besides the application for fish-eye effect and education, in the field of measuring and mapping indoor spaces where accuracy and uniquity is important perspective with six vanishing point may give an effective solution. Different geometrical methods for mapping and curve fitting can be found in the current applications and in the corresponding literature. [8-[10] The advantage of this method is that the measurement is based on only angles and even foreshortenings can be handled. Measurement and control based on angles is already in use in other fields of sciences such as detecting position and orientation of satellites. [11]

Besides since with this method the whole surrounding view can be represented and the representation does not depend on the angle of view there are no partial distortions by mistake. Any part of the image can be selected and knowing the coordinates the original space can be reconstructed. This can give further research topics in the field of mobile robot navigation especially regarding optimalisation for computing techniques [12] and since after practice the image can be expressive and con- tain easily computable data, this method can give an alternative solution for aesthetic markers also [13].

An alternative option for application is to help at 3D graphical softwares for positioning HDRI images Fig. 30 shows three rendered HDRI images. The inaccurate fitting of these images can be detected only after the rendering. [14]

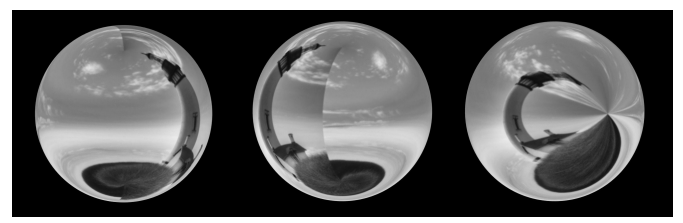

Fig. 30. HDRI images rendered in different positions

Transforming the actual image to a preview in perspective with six vanishing points could help to preset the position and reduce the time of the process.

\section{Conclusions}

The system built up from perspectives based on the number of the vanishing points is completed with the sixth one, in case that the vanishing points are both ideal points of each of the 3 axes of a cartesian coordinate system. Thereby besides the origin of the above mentioned cartesian coordinate system, the whole space can be represented on one image with perspective with six vanishing points.

However the image is not expressive at every part, after creating an image transformations can be done digitally. The mapping method described in this paper satisfies the rules of perspectivic space representation and can be applied in education, measurements, space reconstruction and graphical softwares also.

Besides the authors are keen on contributing in constructing new applications using the system of perspective with six vanishing points.

\section{Acknowledgement}

Authors would like to say special thanks to Dániel Lőrincz, Csaba Szegedi and Lajos Czeglédi who contributed in all earlier stages of this research.

This work is connected to the scientific program of the "Development of quality-oriented and harmonized $R+D+I$ strategy and functional model at BME" project. This project is supported by the New Hungary Development Plan (Project ID: TÁMOP-4.2.1/B-09/1/KMR-2010-0002).

\section{References}

1 Andersen K, THE GEOMETRY OF AN ART: The History of the Mathematical Theory of Perspective from Alberti to Monge, Springer Science+Business Media; New York, 2007, ISBN 0-387-25961-9.

2 O-Connor JJ, Robertson EF, The MacTutor History of Mathematics archive, School of Mathematics and Statistics, University of St Andrews, Scotland, 2015, http://www-history.mcs.st-and.ac.uk/

3 Flocon A, Barré A, Curvilinear perspective - from visual space to the constructed image, University of California Press; California, 1987, ISBN 0-52005979-4. 
4 Crucker F, Manifold Mirrors - The Crossing Paths of the Arts and Mathematics, Cambridge University Press; Cambridge, 2013, ISBN 978-0-52142963-4.

5 www. thermesphere.com Accessed: 28th June 2015.

6 Bölcskei A, Six-point perspectives of different kinds, 2010. Presentation at the 2. Croatian Conference on Geometry and Graphic.

7 Bölcskei A, Int. Sci. Conf. moNGeometrija 2014, Proceedings Vol. 2, In: Pejic $\mathbf{P}$ (ed.), Faculty of Civil engineering and Architecture in Ni- Serbian Society for Geometry and Graphics SUGIG, 2014, pp. 77-86.

8 Paláncz B, Zaletnyik P, Awange RL, Lewis RL, Computational Study of the 3D Affine Transformation, In: Computational Mathematics: Theory, Methods and Applications, New York: Nova Science Publishers, 2011, pp. 279-312.

9 Barsi A, Performing coordinate transformations by artificial neural network, Allgemeine Vermessungs Nachrichten, 108, (2001), 134-137.

10 Paláncz B, Fitting Data with Different Error Models, The Mathematica Journal, 16, (2014), 1-22.

11 Paláncz B, Numeric-Symbolic Solution for Satellite Trajectory Control by Pole Placement, Periodica Polytechnica Civil Engineering, 57(1), (2013), 21-26, DOI 10.3311/PPci.2138

12 Algavri M, Mathkour $\mathbf{H}$, Ramdane $\mathbf{H}$, Alsulaiman $\mathbf{M}$, Comparative study of soft computing techniques for mobile robot navigation in an unknown environment, Computers in Human Behavior, 50, (2015), 42-56, DOI 10.1016/j.chb.2015.03.062

13 Farkas Z, Szekeres K, Korondi P, Aesthetic marker decoding system for indoor robot navigation, IECON 2014 - 40th Annual Conference of the IEEE, In: IECON 2014 - 40th Annual Conference of the IEEE, Industrial Eletronics Society; Dallas, TX, 2014, pp. 2676-2681, DOI 10.1109/IECON.2014.7048884

14 Lörincz D, Szilágyi B, Urbin Á, Space representation with six vanishing points, In: Projecting Spaces - 9th EAEA Conference, EAEA Secretariat, Chair of Visualisation, Faculty of Architecture, Brandenburg University of Technology, 2011, pp. 179-193. 